\title{
Numerical Modeling of two Microwave Sensors for Biomedical Applications §
}

\author{
Xiue Bao $^{1}$ | Giovanni Crupi ${ }^{2 \dagger}$ | llja Ocket ${ }^{1}$ | \\ Juncheng Bao ${ }^{1}$ | Frederik Ceyssens ${ }^{3}$ | Michael $\mathrm{Kraft}^{3}$ \\ | Bart Nauwelaers ${ }^{1}$ | Dominique Schreurs ${ }^{1}$
}

${ }^{1}$ TELEMIC, Electronic Engineering

Department, KU Leuven, B-3001 Leuven, Belgium

${ }^{2}$ BIOMORF Department, University of Messina, 98100 Messina, Italy

${ }^{3}$ MICAS, Electronic Engineering Department, KU Leuven, B-3001 Leuven, Belgium

\section{Correspondence}

Xiue Bao, Electronic Engineering Department, KU Leuven, B-3001 Leuven, Belgium

Email: xiue.bao@esat.kuleuven.be

\section{Funding information}

This work was supported in part by the $\mathrm{KU}$ Leuven under project C24/15/015

Microwave Microbiology ( $\left.\mu^{2} \mathrm{BIO}\right)$, in part by the $\mathrm{KU}$ Leuven under project Hercules, in part by the MTT-S 2018 Graduate Fellowship, and in part by the KU Leuven PDM fellowship.
The purpose of this invited paper is to give readers a critical and comprehensive overview on how to extract dielectric properties of a bioliquid within a broad frequency range. Two sensors are used in the paper to characterize saline solutions by measuring the broadband complex permittivity. The two sensors are based on transmission line and interdigital electrodes designed for low- and high- frequency measurements, respectively, on the basis of the coplanar waveguide structure due to its convenience of fabrication and integration with microfluidic structures for liquid measurements. Different from traditional work where the finite element simulation method is used, the characterization theories of the two sensors are built based on a numerical modeling procedure, which can dramatically increase the device design efficiency, taking just a few seconds. Differently from the finite element method, the proposed numerical analysis utilizes a conformal mapping technique for both sensors. The characterization theories of the two sensors are validated by measuring de-ionized water. The platform is finally used to measure $0.1 \mathrm{~mol} / \mathrm{L}$ and $0.5 \mathrm{~mol} / \mathrm{L}$ saline solutions within a broadband frequency range going from $10 \mathrm{MHz}$ up to $50 \mathrm{GHz}$, with the repeatability error within

§Invited Paper. 
$5 \%$.

\section{KEYWORDS}

Bioengineering, biosensing, conformal mapping, coplanar waveguide, dielectric property, interdigital electrodes, numerical modeling

\section{1 | INTRODUCTION}

Dielectric spectroscopy (DS) is a very powerful investigation technique that is based on studying the frequencydependent dielectric response of matter to applied electromagnetic (EM) fields [1], thereby enabling analysis of the underlying dielectric properties of a wide variety of biological materials under test (MUTs) (e.g., tissue [2], blood [3], proteins [4], viruses [5, 6], and cultured cells [7, 8]). To characterize MUTs and to detect their dielectric properties at microwave frequencies, a plethora of different sensor topologies have been proposed over the years, finding various applications in life science, such as microwave diagnostics in medicine [9, 10,11, 12], and each one with its own pros and cons. Therefore, in choosing the most appropriate microwave sensor, it is essential to identify and prioritize which features of the sensor are the most relevant, depending on the application requirements. Microwave DS offers many advantages over other sensing modalities (e.g., optical, mechanical, and chemical), since this technique is label-free, non-invasive, non-contact, non-expensive, and fast [13]. Given the recent advances in micro- and nanotechnologies, the integration of microwave sensors with microfluidic channels is nowadays experiencing a remarkable progress, enabling microwave dielectric characterization of microliter and even nanoliter volumes of the liquid under test (LUT) $[14,15,16,17]$. The continuous miniaturization of microfluidic sensors is highly demanded for developing lab-on-achip (LoC) technologies [18, 19, 20], which are essential for enabling point-of-care (PoC) devices [21], and in turn their relevant applications (e.g., viral infection detection [22]).

Generally, microwave microfluidic sensors can be classified into two main categories: resonant and non-resonant types. However, the traditional resonant sensors have the drawbacks of requiring a bulky and non-planar structure and of working only in specific frequency bands [23], whereas a broadband dielectric characterization is of crucial importance as the biological fluids exhibit specific dielectric dispersion responses at different frequencies. Therefore, significant attention is paid currently on developing user-friendly and compact sensor platforms for performing broadband dielectric spectroscopy of small fluid volumes. To accomplish this tough task, different solutions have been recently developed by using coplanar waveguide (CPW) technology. As well-known, the integration of CPW transmission lines (TLs) with microfluidic channels allows achieving broadband dielectric spectroscopy [24, 8, 25, 26], but its applicability is limited to relatively high frequencies (generally larger than $100 \mathrm{MHz}$ ). This is because at lower frequencies, it is necessary to use very long TLs that result in a larger space consumption and a larger sample volume. To overcome this drawback, microfluidic platforms based on combining CPW TL and interdigital electrode (IDE) sensors have been proposed, whereby the IDE is a capacitive sensor and is dedicated to the experimental characterization of the LUT in the lower frequency range [27, 8].

Undoubtedly, the most studied liquid is water $\left(\mathrm{H}_{2} \mathrm{O}\right)$, owing to its importance, abundance, and unique properties [28]. As well known, water is the most abundant liquid on Earth and it is of vital importance for all living organisms, which are composed mostly of water. It is worth bearing in mind that about two-thirds of the human body weight is made up of water. Being able to dissolve more substances than any other liquid, water can act as an excellent solvent with which to make aqueous solutions. Among the various water-based liquids, great attention is paid to de-ionized (DI) water and saline aqueous solutions, often simply referred to as saline $[29,30]$. The former is water from which its 
dissolved ions have been removed, while the latter is water in which sodium chloride $(\mathrm{NaCl}$, more commonly known as salt) is dissolved. DI water and saline attract much research interest, because of their wide range of practical applications in different fields, including food, cosmetic, pharmaceutical, and biomedical areas. As an illustrative example, we may consider physiological saline, also known as normal saline, which is essentially isotonic with tissue fluids or blood and thus has a widespread use in medical practice [31, 32].

Within this challenging and stimulating context, the aim of this invited article is to contribute to the advancement of microwave microfluidic sensors by presenting the development of a sensing platform that can be used to perform broadband dielectric spectroscopy measurements on both DI water and saline solutions, as a case study. The proposed microfluidic platform consists of a two-port network based on coupling a CPW TL sensor with an IDE sensor, which are dedicated to high- and low-frequency characterization, respectively. The platform is fabricated on a low-loss quartz substrate and both TL and IDE sensors are integrated with SU-8 microfluidic structures for liquid confinement. To optimize the design of the two sensors and the extraction of the complex permittivity of the LUTs, a numerical modeling procedure is developed by exploiting the numerical conformal-mapping (CM) analysis that is widely used in many research fields $[33,34,35,36,37]$. By using the numerical modeling equations to replace the traditional simulation methods, the designing procedure becomes very time-saving, only taking a few seconds. The broadband experimental characterization is performed from $10 \mathrm{MHz}$ up to $50 \mathrm{GHz}$ by using a conventional vector network analyzer (VNA) to measure the two-port scattering (S-) parameters [38]. For accurate permittivity extraction, calibration techniques are necessarily used [39, 40,41]. By using de-embedding procedures [42], the reference planes of the on-wafer microwave measurements are finally shifted from the probe tips to the sensing area. As a first step, the sensing platform is successfully validated through the good agreement between the measured frequency-dependent complex permittivity of DI water and the data reported in the literature [43]. Afterwards, the broadband-measurement platform is used to analyze the dielectric properties of two saline solutions having $\mathrm{NaCl}$ concentrations of 0.1 and 0.5 $\mathrm{mol} / \mathrm{L}$, as physiological saline is in this concentration range. It is worth noticing that, although the present investigation has been focused on DI water and saline solutions, the developed sensing platform can be straightforwardly used for accomplishing broadband dielectric characterization of various liquids.

This paper is organized as follows: Section II describes the design and fabrication of the microwave measurement platform based on the TL and IDE sensors; Section III illustrates the proposed modelling procedure that is based on the $\mathrm{CM}$ technique; Section IV presents the experimental results concerning the broadband dielectric characterization of DI water and two saline solutions; and, finally, Section $\mathrm{V}$ draws the main conclusions.

\section{2 | DESIGN AND FABRICATION OF MICROWAVE SENSORS FOR BROAD- BAND MEASUREMENTS}

This section is divided into two subsections. The first one is devoted to the design of the two studied sensors, while the second one is dedicated to the description of their fabrication.

\section{1 | Device Design}

For broadband measurements, a CPW TL sensor is firstly designed, as has been schematically shown in Fig. 1. The coplanar waveguide structure is used due to the fact that it is easy to fabricated, especially convenient to integrated with other structures, and it can provide extremely high frequency response. Taking the structures and the dimensions of the ground-signal-ground (GSG) probes for the frequency range of interest, the width of the CPW signal plane, the 


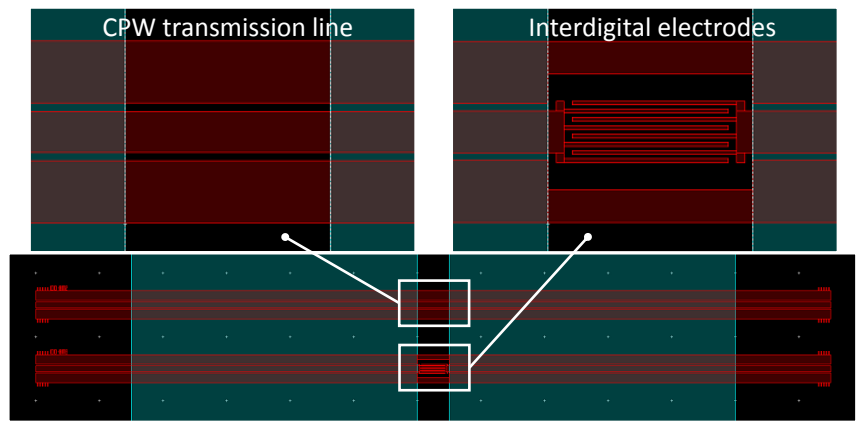

FIGURE 1 Layout of a CPW structure based microfluidic TL sensor and IDE sensor.

ground planes, and the spacing between the signal plane and a ground plane are designed as $100 \mu \mathrm{m}, 150 \mu \mathrm{m}$, and $20 \mu \mathrm{m}$, respectively. As the CPW TL sensor mainly provide accurate measurement results within high frequencies, an IDE sensor is designed for low frequency measurements. As shown in Fig. 1, the IDE sensor is designed based on the CPW structure, using the CPW structure as the feeding lines, which have same cross dimension as the CPW TL sensor. At the sensing area of the IDE based sensor, there are eight electrodes patterned in the middle of the signal plane of a CPW line. The length of the electrodes is $380 \mu \mathrm{m}$. The width of the interdigital electrodes and the spacing between the electrodes are both $10 \mu \mathrm{m}$.

For fluid confinement, an SU-8 microfluidic channel is designed perpendicularly to the sensing electrodes of the two sensors. The choice of SU-8 material as the fluid channel is based on the fact that SU-8 is biocompatible and especially that it can be precisely manufactured. The width of the fluid channel is $500 \mu \mathrm{m}$. The width of the SU-8 covered part and the bare CPW line part at either side of the channel are $4500 \mu \mathrm{m}$ and $1500 \mu \mathrm{m}$, respectively. The thickness of the SU-8 layer is about $400 \mu \mathrm{m}$.

\section{2 | Device Fabrication}

The devices are fabricated in the cleanroom lab of Leuven NanoCentre with the fabrication process described as follows [44]. Due to its transparency, isotropic permittivity, and low loss property, a 4-inch fused silica (quartz) ( $\varepsilon_{r}$ $=3.78, \tan \delta=0.0001$ at $10 \mathrm{GHz}$ and $25^{\circ} \mathrm{C}$ ) wafer, is herein used. Prior to the lithography process, the wafer is submerged in boiling Piranha solution $\left(\mathrm{H}_{2} \mathrm{SO}_{4}: \mathrm{H}_{2} \mathrm{O}_{2}=3: 1\right)$ about 10 minutes for cleaning, which is followed by a dehydration on a $200^{\circ} \mathrm{C}$ hotplate for about 2 minutes. Then, a $45 \mathrm{~nm}$ thick $\mathrm{Cr}$ adhesion layer is sputtered on the $1 \mathrm{~mm}$ thick quartz wafer, on top of which a $500 \mathrm{~nm}$ thick gold layer follows using the sputter deposition technique. After a dehydration procedure, a negative photoresist (ma-N 1410) is spin-coated, followed by a soft-bake at $120^{\circ} \mathrm{C}$ for 90 seconds. Next, the wafer is exposed to ultraviolet (UV) light through a pattern mask, and then developed in the related photoresist developer (ma-D 533) to remove the unexposed part. Following a hard-bake for 3 minutes, the wafer is merged in $3 \mathrm{HCl}: 1 \mathrm{HNO}_{3}: 3 \mathrm{H}_{2} \mathrm{O}$ liquid for 73 seconds and $\mathrm{CR}-14$-used-72X for 18 seconds to define the microwave CPW electrodes.

After obtaining the wafer with patterned Cr/Au electrodes, a $350 \mu \mathrm{m}$ thick SU-8 film is defined. First, about a $1 \mu \mathrm{m}$ thick layer of MicroChem OmniCoat is spin-coated on the wafer, followed by a soft-bake. Then, 6 gram of SU-8 2005 is poured and evenly spread on the wafer that is balanced on a flat plate. The SU-8 2005 (45\% solids) is obtained by 


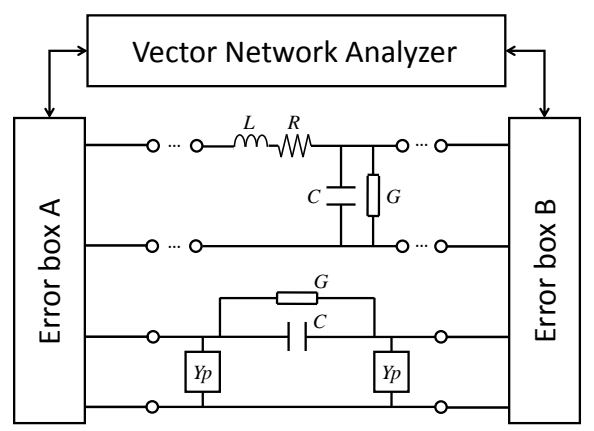

FIGURE 2 Equivalent circuits of a CPW based TL sensor (top) and a series IDE sensor (bottom), where the effects of cables, connectors, connections between probes and the device, and test fixtures are modeled as 'Error box A' and 'Error box B'.

dissolving SU-8 2050 (71.65\% solids) into the organic solvent SU-8 2000 thinner (MicroChem. Corp.). Next, the wafer is baked at $80^{\circ} \mathrm{C}$ on a hotplate for 24 hours, in order to partially evaporate the solvent content before exposure [37]. Afterwards, the SU-8 channel layer is patterned by exposing to near-UV light through a mask, followed by post-baking, and is then developed in a propylene-glycol-methyl-ether-acetate (PGMEA) developer (MicroChem. Corp.) for about 80 seconds.

\section{I CAPACITANCE AND CONDUCTANCE MODELING}

During the measurements, the two devices illustrated in Fig. 1 are connected to a VNA by cables and connectors. The cables, connectors, connections between probes and the device, and the feeding part (including the bare line part and the SU-8 covered feeding line part) are modeled together as the "Error box A" and the "Error box B", presented in Fig. 2. The equivalent circuit at the sensing area of the two coplanar sensors are also shown in Fig. 2. The rest of this section is divided into two parts, which are focused on the modeling of the CPW TL and IDE sensors, respectively.

\section{1 | Modeling of the CPW Transmission Line}

For the CPW TL, we can assume that a quasi-TEM mode is propagating throughout the line. The coplanar electrodes are assumed to be sandwiched between the dielectric substrate $\left(\varepsilon_{s u b}, \tan \delta_{s u b}\right)$ and a MUT $\left(\varepsilon_{r}^{*}=\varepsilon_{r}^{\prime}-j \varepsilon_{r}^{\prime \prime}\right.$, with $\varepsilon_{r}^{\prime \prime}=\varepsilon_{r}^{\prime} \cdot \tan \delta$ ) [45]. As shown in Fig. 2, the sensing area of the coplanar TL is modeled as an infinite cascade of twoport elementary sections, each representing an infinitesimally short segment of the line. Each elementary section consists of four frequency-dependent distributed per unit length (p.u.l.) parameters - resistance $R(\omega)$, inductance $L(\omega)$, capacitance $C(\omega)$, and conductance $G(\omega)$. The resistance $R$ and the inductance $L$ are independent of the dielectric material on either side of the CPW electrodes, and are only determined by the signal and ground conductors $[24,8]$.

According to the conformal mapping technique and multilayer theory $[45,17]$, the distributed p.u.l $C$ and $G$ can 
be expressed as [37]

$$
\begin{aligned}
& C=\varepsilon_{0}\left[2 \frac{K\left(k_{0}\right)}{K\left(k_{0}^{\prime}\right)}+\left(\varepsilon_{s u b}-1\right) \frac{K\left(k_{s u b}\right)}{K\left(k_{s u b}^{\prime}\right)}+\left(\varepsilon_{r}^{\prime}-1\right) \frac{K\left(k_{r}\right)}{K\left(k_{r}^{\prime}\right)}\right] \\
& G=\omega \varepsilon_{0}\left[\varepsilon_{s u b} \tan \delta_{s u b} \frac{K\left(k_{s u b}\right)}{K\left(k_{s u b}^{\prime}\right)}+\varepsilon_{r}^{\prime \prime} \frac{K\left(k_{r}\right)}{K\left(k_{r}^{\prime}\right)}\right]
\end{aligned}
$$

where $\varepsilon_{0}=8.8542 \cdot 10^{-12} \mathrm{~F} / \mathrm{m}$ is the vacuum permittivity, $K(k)$ is the complete elliptic integral of the first kind, and $K\left(k^{\prime}\right)=K\left(\sqrt{1-k^{2}}\right)$. The elliptic moluli $k_{0}$ and $k_{i}(i=s u b, r)$ are calculated with the following equations [37]

$$
\begin{aligned}
& k_{0}=\frac{w}{w+2 s} \sqrt{\frac{1-(w+2 s)^{2} /(w+2 s+2 g)^{2}}{1-w^{2} /(w+2 s+2 g)^{2}}} \\
& k_{i}=\frac{\sinh \left(\frac{\pi w}{4 h_{i}}\right)}{\sinh \left(\frac{\pi(w+2 s)}{4 h_{i}}\right)} \sqrt{\frac{1-\sinh ^{2}\left(\frac{\pi(w+2 s)}{4 h_{i}}\right) / \sinh ^{2}\left(\frac{\pi(w+2 s+2 g}{4 h_{i}}\right)}{1-\sinh ^{2}\left(\frac{\pi w}{4 h_{i}}\right) / \sinh ^{2}\left(\frac{\pi(w+2 s+2 g}{4 h_{i}}\right)}}
\end{aligned}
$$

where $h_{i}(i=s u b, r)$ is the thickness of the substrate or the MUT; $w, g$, and $s$ are half of the signal conductor width, half of the finite ground width, and half of the spacing between the signal conductor and the ground of the CPW line, respectively. Therefore, according to the expressions of (1) to (4), once the dimensions and substrate are determined, the TL $C$ is linearly related to the MUT's real part permittivity $\varepsilon_{r}^{\prime}$, and similarly, the TL $G / \omega$ is linearly dependent on the MUT's imaginary part permittivity $\varepsilon_{r}^{\prime \prime}$. The linear relationships form the foundation of extracting the MUT complex permittivity using the TL method. Noticeably, further comparison of (1) and (2) indicates that $\varepsilon_{r}^{\prime}$ and $\varepsilon_{r}^{\prime \prime}$ have the same coefficient $\frac{K\left(k_{r}\right)}{K\left(k_{r}^{\prime}\right)}$, which can be defined as the sensitivity [8] of the TL sensor. In our experience, with a known substrate, a larger $w /(w+s)$ ratio is associated with a higher measurement sensitivity for the complex permittivity extraction.

The TL capacitance $C$ and the conductance $G$ can also be extracted using the following expression [40]

$$
G(\omega)+j \omega C(\omega)=\frac{\gamma}{Z_{c}}
$$

where $Z_{c}$ and $\gamma$ are the line characteristic impedance and the propagation constant, respectively. They can be obtained from the following chain matrix, which can be readily obtained by performing a transformation on the Sparameters of the MUT loaded TL [40].

$$
\left[\begin{array}{ll}
A & B \\
C & D
\end{array}\right]=\left[\begin{array}{cc}
\cosh (\gamma /) & Z_{c} \cdot \sinh (\gamma /) \\
\frac{1}{Z_{c}} \cdot \sinh (\gamma /) & \cosh (\gamma /)
\end{array}\right]
$$

with / the length of the TL. Therefore, based on the above analysis, the dielectric properties of the material under observation can be accurately determined from the S-parameters of the material loaded TL. Additionally, with given substrate, regardless of the TL dimensions and the dielectric properties of the MUT, the capacitance $C$ is very sensitive to $h_{r}$ when the MUT thickness $h_{r}<(w+2 s)$, whereas the capacitance $C$ is almost constant when $h_{r} \geqslant(w+2 s)$. This means that when the CPW line works as a sensor, the thickness of the to-be-tested material should be larger than $(w+2 s)$ to reduce the measurement uncertainty related to the electromagnetic field distribution. 


\subsection{Modeling of the IDE Structure}

As the IDE based sensor is intended to work at low RF/microwave frequencies, where the wavelength is very large compared to the dimension of the sensing electrodes, the electrodes in the sensing area can be accurately modeled as a lumped circuit [27]. The equivalent circuit of the series IDE is presented in Fig. 2. The sensing area of the IDE is modelled as a capacitance $C$ in parallel with a conductance $G$. It should be noticed that $Y_{p}$ in Fig. 2 results from the slots between the CPW signal plane and the CPW ground planes and is assumed to be independent on the MUT. The capacitance $C$ and conductance $G$ in the sensing area of the IDE can be calculated using the following equations [46]

$$
\begin{aligned}
& C=L \cdot\left[(n-3) \frac{C_{I}}{2}+2 C_{E}\right] \\
& G=L \cdot\left[(n-3) \frac{G_{I}}{2}+2 G_{E}\right]
\end{aligned}
$$

where $L$ is the length of the interdigital electrodes and $n$ is the total number of electrodes. $C_{I}$ and $G_{I}$ are the p.u.l. capacitance and the p.u.l. conductance between any two interior and adjacent electrodes of the IDE sensing area. $C_{E}$ and $G_{E}$ are the p.u.l. capacitance and the p.u.I. conductance for the two exterior and adjacent electrodes of the IDE sensing area. Based on the conformal mapping technique, $C_{E}$ and $G_{E}$ can be calculated with [46]

$$
\begin{aligned}
& C_{E}=\varepsilon_{0}\left[2 \frac{K\left(k_{E 0}\right)}{K\left(k_{E 0}^{\prime}\right)}+\left(\varepsilon_{s u b}-1\right) \frac{K\left(k_{E s u b}\right)}{K\left(k_{E s u b}^{\prime}\right)}+\left(\varepsilon_{r}^{\prime}-1\right) \frac{K\left(k_{E r}\right)}{K\left(k_{E r}^{\prime}\right)}\right] \\
& G_{E}=\omega \varepsilon_{0}\left[\varepsilon_{s u b} \tan \delta_{s u b} \frac{K\left(k_{E s u b}\right)}{K\left(k_{E s u b}^{\prime}\right)}+\varepsilon_{r}^{\prime \prime} \frac{K\left(k_{E r}\right)}{K\left(k_{E r}^{\prime}\right)}\right]
\end{aligned}
$$

in which the elliptic moduli $k_{E O}$ and $k_{E i}$ are expressed in terms of the electrodes' dimensions, as

$$
\begin{aligned}
& k_{E 0}=\frac{w_{f}}{w_{f}+2 s_{e}} \sqrt{\frac{1-\left(w_{f}+2 s_{e}\right)^{2} /\left(w_{f}+2 s_{e}+w_{e}\right)^{2}}{1-w_{f}^{2} /\left(w_{f}+2 s_{e}+w_{e}\right)^{2}}} \\
& k_{E i}=\frac{\sinh \left(\frac{\pi w_{f}}{2 h_{i}}\right)}{\sinh \left(\frac{\pi\left(w_{f}+2 s_{e}\right)}{2 h_{i}}\right)} \cdot \sqrt{\frac{1-\sinh ^{2}\left(\frac{\pi\left(w_{f}+2 s_{e}\right)}{2 h_{i}}\right) / \sinh ^{2}\left(\frac{\pi\left(w_{f}+2 s_{e}+w_{e}\right.}{2 h_{i}}\right)}{1-\sinh ^{2}\left(\frac{\pi w_{f}}{2 h_{i}}\right) / \sinh ^{2}\left(\frac{\pi\left(w_{f}+2 s_{e}+w_{e}\right.}{2 h_{i}}\right)}}
\end{aligned}
$$

where $w_{f}$ is half of the interior electrodes' width, $w_{e}$ is the width of the exterior two electrodes, and $s_{e}$ is half of the space between an interior electrode and an exterior electrode [30]. The p.u.l. capacitance $C_{I}$ formed by all interior unit cells and the interior p.u.l. conductance $G_{I}$ are calculated by [46]

$$
\begin{aligned}
& C_{I}=\varepsilon_{0}\left[2 \frac{K\left(k_{I 0}\right)}{K\left(k_{I 0}^{\prime}\right)}+\left(\varepsilon_{\text {sub }}-1\right) \frac{K\left(k_{I s u b}\right)}{K\left(k_{I s u b}^{\prime}\right)}+\left(\varepsilon_{r}^{\prime}-1\right) \frac{K\left(k_{I r}\right)}{K\left(k_{I r}^{\prime}\right)}\right] \\
& G_{I}=\omega \varepsilon_{0}\left[\varepsilon_{\text {sub }} \tan \delta_{\text {sub }} \frac{K\left(k_{I s u b}\right)}{K\left(k_{I s u b}^{\prime}\right)}+\varepsilon_{r}^{\prime \prime} \frac{K\left(k_{I r}\right)}{K\left(k_{I r}^{\prime}\right)}\right]
\end{aligned}
$$

where the expressions of moduli $k_{I 0}$ and $k_{I i}$ are

$$
\begin{aligned}
& k_{I 0}=\cos \left(\frac{\pi s_{f}}{2\left(w_{f}+s_{f}\right)}\right) \\
& k_{I i}=\sqrt{\frac{1}{t_{3}}}
\end{aligned}
$$


where $s_{f}$ is half of the space between two interior electrodes, and $t_{3}$ is calculated with

$$
t_{3}=\frac{\left(r_{1}-r_{3}\right)\left(r_{4}-r_{2}\right)}{\left(r_{4}-r_{3}\right)\left(r_{1}-r_{2}\right)}
$$

by which $r_{1}, r_{2}, r_{3}$, and $r_{4}$ can be obtained using the following Jacobi elliptic function, respectively corresponding with $z_{1}=0, z_{2}=\frac{w_{f}}{w_{f}+s_{f}} K\left(k_{x i}\right), z_{3}=K\left(k_{x i}\right)$, and $z_{4}=K\left(k_{x i}\right)+j K\left(k_{x i}^{\prime}\right)[46]$

$$
r=s n\left(z, \frac{\theta_{2}^{2}\left(e^{-\pi \frac{w_{f}+s_{f}}{h_{i}}}\right)}{\theta_{3}^{2}\left(e^{-\pi \frac{w_{f}+s_{f}}{h_{i}}}\right)}\right)
$$

where $\theta_{2}$ and $\theta_{3}$ are the Jacobi theta functions [47]. According to (7)-(18), once the dimensions and the substrate of the IDE are determined, $C$ and $G$ are linearly dependent on both the real and imaginary parts of the permittivity of the MUT loaded on top of the interdigital electrodes [17, 8].

The equivalent capacitance $C$ and conductance $G$ of the IDE sensor can also be readily obtained by [27]

$$
G+j \omega C=-\left(Y_{12}+Y_{21}\right) / 2
$$

As the tested device is reciprocal, the admittance parameter $Y_{12}$ should be equal to $Y_{21}$, but their average $\left(Y_{12}+Y_{21}\right) / 2$ is used to estimate $G$ and $C$, in order to reduce measurement uncertainties. The values of $Y_{12}$ and $Y_{21}$ can be extracted from the S-parameters of the series IDE sensing area using the following equations [40]

$$
\begin{aligned}
& Y_{12}=Y_{0} \frac{-2 S_{12}}{\left(1+S_{11}\right)\left(1+S_{22}\right)-S_{12} S_{21}} \\
& Y_{21}=Y_{0} \frac{-2 S_{21}}{\left(1+S_{11}\right)\left(1+S_{22}\right)-S_{12} S_{21}}
\end{aligned}
$$

where $Y_{0}=1 / Z_{0}$ and $Z_{0}$ is the reference impedance of the measurement system. Thus, once the S-parameters of the MUT loaded IDE are obtained, the complex permittivity of the material can be easily extracted.

\section{4 | BROADBAND MEASUREMENTS OF LIQUIDS}

This section is organized in two subsections: the former is aimed at describing the calibration and measurement procedure based on using the proposed sensing platform, while the latter is focused on analyzing the broadband experimental results achieved for DI water and two saline solutions.

\section{1 | Calibration and Measurements}

When performing the measurements, the microfluidic devices are connected with two probes, which are connected to the Agilent E8361A VNA with cables and connectors. In order to characterize the dielectric MUT loaded on the sensing area of the two sensing devices, an accurate calibration procedure is essential. As shown in Fig. 2, the errors caused by the connectors, connections between probes and the chip, cables, and the feeding test fixture parts between the VNA and the sensing area are modeled as "Error box A" and "Error box B" for the left part and the right part of the sensing area, respectively. According to the design description of the two sensing devices in Section II, "Error 
box A" and "Error box B" are assumed to be the same for both the TL sensor and the IDE sensor.

For high frequencies (>100 MHz), the multiline thru-reflect-line (TRL) calibration method [39] can be used to move accurately the reference planes to the probe-tips as the first-step calibration. A series of multiline TRL calibration standards are fabricated on the same wafer as the two sensors to minimize fabrication variations. The cross section of the standards are the same as the air-covered feeding part of the sensor as shown in Fig. 1, and their lengths are $0.5 \mathrm{~mm}$ (Thru), $0.25 \mathrm{~mm}$ (Short), $1 \mathrm{~mm}$ (Line 1), $3 \mathrm{~mm}$ (Line 2), $7 \mathrm{~mm}$ (Line 3), $10 \mathrm{~mm}$ (Line 4), and $18 \mathrm{~mm}$ (Line 5), respectively.

Next, to obtain accurately the dielectric properties of the material loaded in the sensing area, the reference planes have to be further moved from the probe tips to the sensing area with a second-step calibration [42], removing the errors resulting from the air-covered and SU-8 covered feeding parts on both sides of the sensing area. At high frequencies, the air-covered and SU-8 covered feeding parts are both modeled as distributed TL segments [27]. The characteristic impedance and propagation constant of the air-covered feeding part are assumed to be $Z_{\text {air }}$ and $\gamma_{a i r}$, and the characteristic impedance and propagation constant of the SU-8-covered feeding part are denoted by $Z_{s u-8}$ and $\gamma_{s u-8}$. The parameters $Z_{\text {air }}, \gamma_{a i r}, Z_{s u-8}$, and $\gamma_{s u-8}$ can be obtained by measuring a bare line and an SU-8-covered line, which have the same cross-section as the feeding line part and whose lengths are known [27]. Then using (6), the chain matrices of the air-covered and the SU-8 covered feeding segments are readily obtained. The relationship of the complete TL sensor within the two probe-tips, the left/right bare feeding line, left/right SU-8-covered feeding line, and the sensing area covered with MUT, can be described, using their respective chain matrices, as the following equation

$$
\left[\begin{array}{ll}
A & B \\
C & D
\end{array}\right]_{\text {tot }}=\left[\begin{array}{ll}
A & B \\
C & D
\end{array}\right]_{\text {air } L} \cdot\left[\begin{array}{ll}
A & B \\
C & D
\end{array}\right]_{s u-8 L} \cdot\left[\begin{array}{ll}
A & B \\
C & D
\end{array}\right]_{\text {mut }} \cdot\left[\begin{array}{ll}
A & B \\
C & D
\end{array}\right]_{s u-8 R} \cdot\left[\begin{array}{ll}
A & B \\
C & D
\end{array}\right]_{\text {air } R} .
$$

Consequently, the reference planes are moved to the sensing area, and the chain matrix of the MUT loaded TL can be determined by matrix operations. Using the extraction process that is described in Section III, the frequency dependent permittivity of the material loaded on the TL sensing area can be readily calculated.

For low frequency measurements $(<1 \mathrm{GHz})$, the first-step calibration is performed on the commercial impedance standard substrate (ISS) CS-5 (GGB Industries, Inc.) using the line-reflect-match (LRM) calibration method to move the reference planes from the VNA ports to the probe-tips. Then, the feeding line, which consists of the air-covered and the SU-8-covered parts, is modeled as "de-embedding". The "de-embedding" applies to both sides of the IDE sensor due to its symmetrical structure. Therefore, the de-embedding procedure can be summarized as

$$
T_{\text {tot }}=T_{d e-e m} \cdot T_{m u t} \cdot T_{d e-e m}^{-1}
$$

where $T_{d e-e m}$ is the wave-cascade matrix of the error box "de-embedding", $T_{\text {mut }}$ is the wave-cascade matrix of the sensing area loaded with MUT, and $T_{\text {tot }}$ is the wave-cascade matrix of the whole low frequency sensor between the two probe-tips. The transformation from the scattering parameters to the wave-cascade matrix is defined by [40]

$$
T=\frac{1}{S_{21}}\left[\begin{array}{cc}
S_{12} S_{21}-S_{11} S_{22} & S_{11} \\
-S_{22} & 1
\end{array}\right]
$$

By directly measuring the de-embedding standard that has the same air-covered and SU-8 covered structure and dimensions, $T_{d e-e m}$ is readily measured, and consequently $T_{m u t}$ can be extracted. $T_{d e-e m}$ can also be obtained by extracting accurate distributed parameters of the feeding TL using the procedure above described, but it involves 
a complex first-step calibration [30], which is not necessary for low frequency measurements. After reaching, by matrix transformations, the S-parameters of the $T_{m u t}$, the dielectric properties of the MUT at low frequencies can be characterized with the method described in Section III.

\section{2 | Results and Analysis}

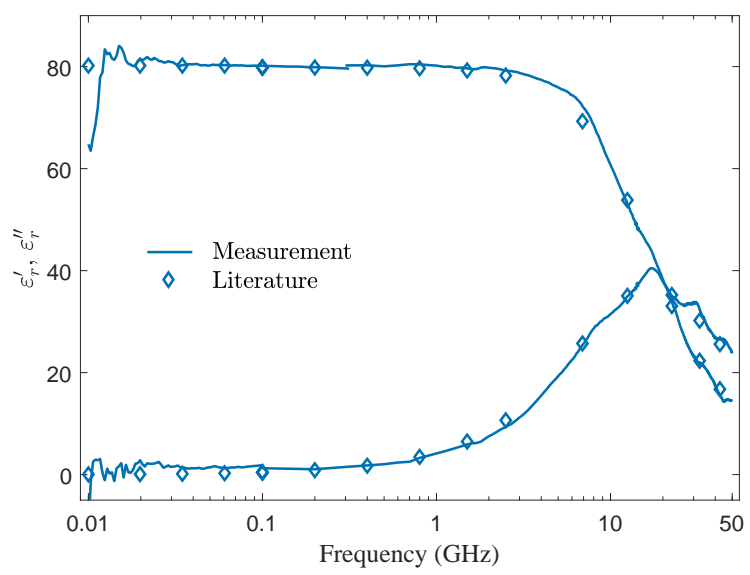

FIGURE 3 The complex permittivity of de-ionzed water measured with the proposed platform that integrates a CPW TL sensor and an interdigital electrode sensor. Literature values [43] are also presented here for reference.

In order to validate the characterization theories provided in previous sections, measurements were first performed on de-ionized water. For low frequency measurements, the IDE sensor was used, sweeping the frequency from $10 \mathrm{MHz}$ to $5 \mathrm{GHz}$. For high frequency measurements, the CPW TL sensor was used with frequency range from $100 \mathrm{MHz}$ to $50 \mathrm{GHz}$. A total of 201 frequency points in a log scale and 501 frequency points in a linear scale were recorded for the low- and high-frequency measurements, respectively. During all the measurements, the VNA power was set at $-20 \mathrm{dBm}$ and the intermediate frequency (IF) bandwidth was $20 \mathrm{~Hz}$. Additionally, beneath the microfluidic wafer, there is an $8 \mathrm{~mm}$ polymethylmethacrylate(PMMA) spacer placed on the surface of the VNA probe station to reduce undesired mode coupling especially at high frequencies. The room temperature was kept at about $20^{\circ} \mathrm{C}$ considering the obvious temperature dependence of the dielectric properties of liquids.

Using the data processing methods described in previous sections, the complex permittivity of de-ionized water was calculated. Fig. 3 reports the measurement results with the IDE sensor from $10 \mathrm{MHz}$ to about $300 \mathrm{MHz}$ and the CPW TL sensor from around $300 \mathrm{MHz}$ to $50 \mathrm{GHz}$. To present the low frequency results more clearly, the measurement values are presented in the log frequency scale. As shown in Fig. 3, the overlap between the two groups of measurement results is very good, confirming the validity of the developed investigation. It should be noticed that the permittivity measurements of de-ionized water were repeated 5 times, with the repeatability error within $5 \%$. The complex permittivity values plotted with the diamond mark in Fig. 3, are calculated from permittivity expressions in [43]. Good agreement between measurement results and literature values on de-ionized water can be clearly seen in Fig. 3 for both sensors, which validates that the proposed measurement platform can provide accurate dielectric permittivity results for liquids.

Next, the platform is used for dielectric spectroscopy measurements of two types of saline solutions using the 


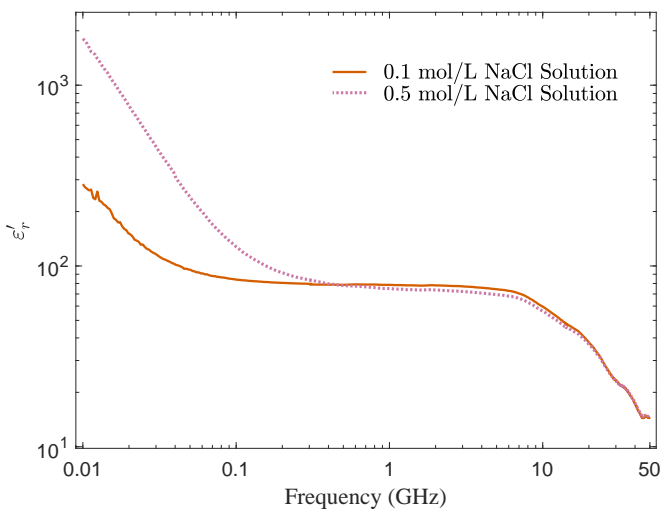

(a)

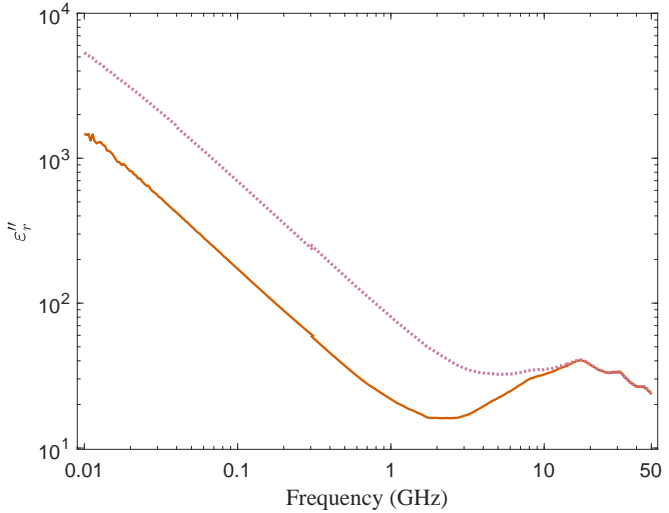

(b)

FIGURE 4 The real (a) and imaginary parts (b) of the permittivity of two $\mathrm{NaCl}$ solutions measured with the proposed platform that integrates a CPW TL sensor and an interdigital electrode sensor.

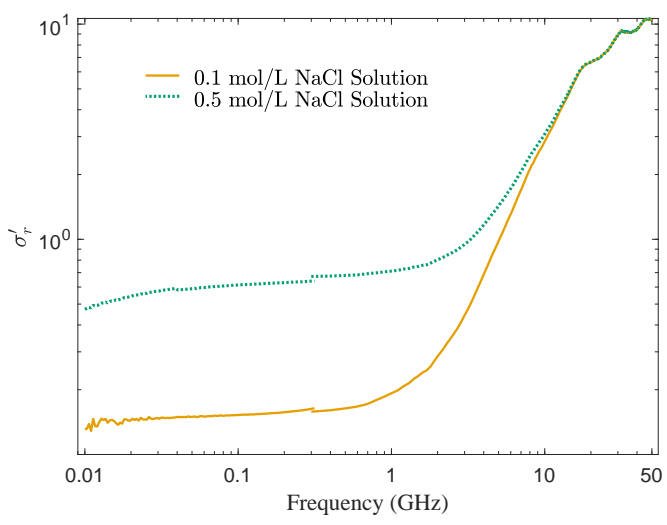

(a)

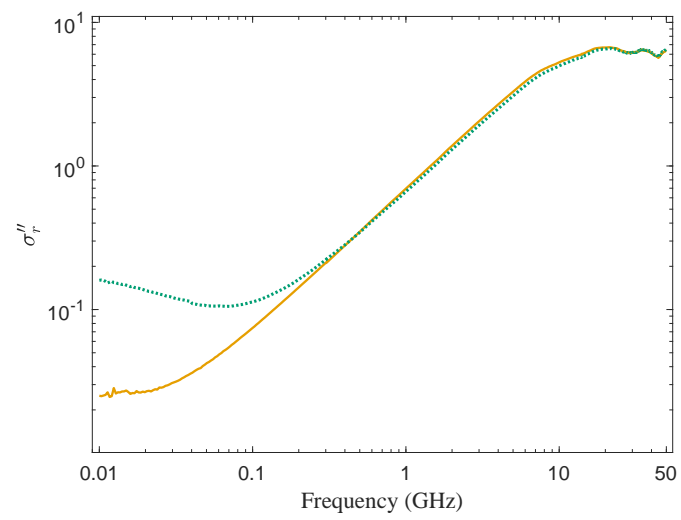

(b)

FIGURE 5 The real (a) and imaginary parts (b) of the conductivity of two saline solutions calculated from the corresponding complex permittivity with $\sigma_{r}^{\prime}+j \sigma_{r}^{\prime \prime}=j \omega \varepsilon_{0} \cdot\left(\varepsilon_{r}^{\prime}-j \varepsilon_{r}^{\prime \prime}\right)$ [30].

same data analysis procedure as for the de-ionized water. The two saline solutions are $0.1 \mathrm{~mol} / \mathrm{L}$ and $0.5 \mathrm{~mol} / \mathrm{L}$ as physiological saline solution (normal saline) is in this concentration range. The measurement results are presented in Fig. 4. A preliminary conclusion can be drawn that higher $\mathrm{NaCl}$ concentration results in larger real and imaginary parts of the permittivity at low frequencies. The ion content in the solutions can result in electrode polarization, which can dramatically affect the analysis of the actual dielectric information of ionic liquids. Consequently, in order to analyze the electrode polarization in the saline solutions, the measured complex permittivity was transformed to complex conductivity with $\sigma_{r}^{\prime}+j \sigma_{r}^{\prime \prime}=j \omega \varepsilon_{0} \cdot\left(\varepsilon_{r}^{\prime}-j \varepsilon_{r}^{\prime \prime}\right)$ [30]. The calculated complex conductivity values are presented in Fig. 5. According to the real part conductivity results in Fig. 5(a), we can directly read out the static conductivity of the solutions in the low frequency range. The read-out static conductivity of the $0.1 \mathrm{~mol} / \mathrm{L}$ saline and the $0.5 \mathrm{~mol} / \mathrm{L}$ saline solutions are about 0.15 and 0.63 , respectively. The plots of the real part conductivity values of the two solutions 
at low frequencies show a slight frequency dependency. This means that the actual complex permittivity of the two solutions at low frequencies is frequency dependent, but unfortunately hidden by the dramatic values caused by the electrode polarization. By observing the plots in Fig. 5(b), the frequency range over which the electrode polarization is dominating can be determined [30]. The electrode polarization frequency ranges for the $0.1 \mathrm{~mol} / \mathrm{L}$ and the $0.5 \mathrm{~mol} / \mathrm{L}$ saline solutions are from very low frequency up to around $25 \mathrm{MHz}$ and $70 \mathrm{MHz}$, respectively.

\section{5 | CONCLUSION}

A compact and user-friendly platform that consists of a CPW TL sensor and an IDE sensor, is proposed. The two sensors are integrated with microfluidic structures using photolithographic micro-device fabrication techniques, making it possible to perform accurate measurements by using a very small volume of sample. A numerical modeling procedure, based on the conformal mapping technique, is applied to both sensors, which leads to the characterization foundation for the dielectric property of the material that is loaded on top of the sensing area of the two sensors. By using the numerical method to replace the traditional simulation method, only a few seconds are needed for the optimization of device design. The platform is successfully validated by measuring the complex permittivity of de-ionized water within a broadband frequency range. Then, the proposed method is used to analyze the dielectric properties of two types of saline solutions. Although the reported experiments have been limited to water-based liquids, the developed microfluidic sensing platform can be readily used for carrying out broadband dielectric spectroscopy measurements on various liquids.

\section{Acknowledgements}

The authors would like to acknowledge all of the staff in the Leuven NanoCentre for the practical instruction in device fabrication.

\section{REFERENCES}

[1] Kremer F. Dielectric spectroscopy-yesterday, today and tomorrow. Journal of Non-Crystalline Solids 2002;305(1-3):19.

[2] Gabriel C, Gabriel S, Corthout yE. The dielectric properties of biological tissues: I. Literature survey. Physics in Medicine \& Biology 1996;41(11):2231.

[3] Facer G, Notterman DA, Sohn L. Dielectric spectroscopy for bioanalysis: From $40 \mathrm{~Hz}$ to $26.5 \mathrm{GHz}$ in a microfabricated wave guide. Applied Physics Letters 2001;78(7):996-998.

[4] Yokoyama K, Kamei T, Minami H, Suzuki M. Hydration study of globular proteins by microwave dielectric spectroscopy. The Journal of Physical Chemistry B 2001;105(50):12622-12627.

[5] Yang SC, Lin HC, Liu TM, Lu JT, Hung WT, Huang YR, et al. Efficient structure resonance energy transfer from microwaves to confined acoustic vibrations in viruses. Scientific Reports 2015;5:18030.

[6] Sun CK, Tsai YC, Chen YJE, Liu TM, Chen HY, Wang HC, et al. Resonant Dipolar Coupling of Microwaves with Confined Acoustic Vibrations in a Rod-shaped Virus. Scientific Reports 2017;7(1):1-9.

[7] Asami K. Characterization of biological cells by dielectric spectroscopy. Journal of Non-Crystalline Solids 2002;305(13):268-277. 
[8] Bao X, Ocket I, Bao J, Doijen J, Zheng J, Kil D, et al. Broadband dielectric spectroscopy of cell cultures. IEEE Transactions on Microwave Theory and Techniques 2018;66(12):5750-5759.

[9] Nikolova NK. Microwave imaging for breast cancer. IEEE Microwave Magazine 2011;12(7):78-94.

[10] Chandra R, Zhou H, Balasingham I, Narayanan RM. On the opportunities and challenges in microwave medical sensing and imaging. IEEE transactions on biomedical engineering 2015;62(7):1667-1682.

[11] Yang X, Shah SA, Ren A, Zhao N, Zhang Z, Fan D, et al. Freezing of gait detection considering leaky wave cable. IEEE Transactions on Antennas and Propagation 2019;67(1):554-561.

[12] Yang X, Fan D, Ren A, Zhao N, Alam M. 5G-based user-centric sensing at C-band. IEEE Transactions on Industrial Informatics 2019;15(5):3040-3047.

[13] Artis F, Chen T, Chretiennot T, Fournie JJ, Poupot M, Dubuc D, et al. Microwaving biological cells: Intracellular analysis with microwave dielectric spectroscopy. IEEE Microwave Magazine 2015;16(4):87-96.

[14] Shaforost E, Klein N, Vitusevich S, Barannik A, Cherpak N. High sensitivity microwave characterization of organic molecule solutions of nanoliter volume. Applied Physics Letters 2009;94(11):112901.

[15] Bao X, Zhang M, Ocket I, Bao J, Kil D, Liu Z, et al. Integration of Interdigitated Electrodes in Split-Ring Resonator for Detecting Liquid Mixtures. IEEE Transactions on Microwave Theory and Techniques 2020;68(6):2080-2089.

[16] née Haase NM, Fuge G, Trieu HK, Zeng AP, Jacob AF. Miniaturized transmission-line sensor for broadband dielectric characterization of biological liquids and cell suspensions. IEEE Transactions on Microwave Theory and Techniques 2015;63(10):3026-3033.

[17] Bao X, Ocket I, Crupi G, Schreurs D, Bao J, Kil D, et al. A planar one-port microwave microfluidic sensor for microliter liquids characterization. IEEE Journal of Electromagnetics, RF and Microwaves in Medicine and Biology 2018;2(1):1017.

[18] Daw R, Finkelstein J, Lab on a chip. Nature Publishing Group; 2006.

[19] Abgrall P, Gue A. Lab-on-chip technologies: making a microfluidic network and coupling it into a complete microsystem-a review. Journal of Micromechanics and Microengineering 2007;17(5):R15.

[20] Dehghan Manshadi MK, Khojasteh D, Mohammadi M, Kamali R. Electroosmotic micropump for lab-on-a-chip biomedical applications. International Journal of Numerical Modelling: Electronic Networks, Devices and Fields 2016;29(5):845858.

[21] Nguyen T, Zoëga Andreasen S, Wolff A, Duong Bang D. From lab on a chip to point of care devices: The role of open source microcontrollers. Micromachines 2018;9(8):403.

[22] Yeh YT, Nisic M, Yu X, Xia Y, Zheng SY. Point-of-care microdevices for blood plasma analysis in viral infectious diseases. Annals of Biomedical Engineering 2014;42(11):2333-2343.

[23] Mohd Bahar AA, Zakaria Z, Md Arshad MK, Alahnomi RA, Abu-Khadrah Al, Sam WY. Microfluidic biochemical sensor based on circular SIW-DMS approach for dielectric characterization application. International Journal of RF and Microwave Computer-Aided Engineering 2019;29(9):e21801.

[24] Booth JC, Orloff ND, Mateu J, Janezic M, Rinehart M, Beall JA. Quantitative permittivity measurements of nanoliter liquid volumes in microfluidic channels to $40 \mathrm{GHz}$. IEEE Transactions on Instrumentation and Measurement 2010;59(12):3279-3288.

[25] Seo S, Stintzing T, Block I, Pavlidis D, Rieke M, Layer PG. High frequency wideband permittivity measurements of biological substances using coplanar waveguides and application to cell suspensions. In: 2008 IEEE MTT-S International Microwave Symposium Digest Atlanta, GA, USA; 2008. p. 915-918. 
[26] Liu S, Ocket I, Cauwe M, Schreurs D, Nauwelaers B. Sensitivity analysis of broadband on-wafer dielectric spectroscopy of yeast cell suspensions up to $110 \mathrm{GHz}$. IEEE Microwave and Wireless Components Letters 2015;25(3):199-201.

[27] Bao X, Ocket I, Bao J, Kil D, Brancato L, Nauwelaers B. Broadband dielectric spectroscopy measurements of liquids combining interdigital capacitor and coplanar waveguide. In: 47th IEEE European Microwave Conference (EuMC) Nuremberg, Germany; Oct. 2017. p. 946-949.

[28] Pettersson LGM, Henchman RH, Nilsson A, Water The Most Anomalous Liquid. ACS Publications; 2016.

[29] Ruvio G, Vaselli M, Lopresto V, Pinto R, Farina L, Cavagnaro M. Comparison of different methods for dielectric properties measurements in liquid sample media. International Journal of RF and Microwave Computer-Aided Engineering 2018;28(3):e21215.

[30] Bao X, Liu S, Ocket I, Liu Z, Schreurs DM, Nauwelaers BK. A modeling procedure of the broadband dielectric spectroscopy for ionic liquids. IEEE Transactions on NanoBioscience 2018;17(4):387-393.

[31] Awad S, Allison SP, Lobo DN. The history of 0.9\% saline. Clinical Nutrition 2008;27(2):179-188.

[32] Ortiz ML, González-Castro A, Peñasco YM, Escudero PA, Chicote EÁ, Jiménez AA, et al. Saline solutions in history. Emergencias: revista de la Sociedad Espanola de Medicina de Emergencias 2019;31(1):58-61.

[33] Ghione G, Naldi CU. Coplanar waveguides for MMIC applications: Effect of upper shielding, conductor backing, finite-extent ground planes, and line-to-line coupling. IEEE Transactions on Microwave Theory and Techniques 1987;35(3):260-267.

[34] Teppati V, Goano M, Ferrero A. Conformal-mapping design tools for coaxial couplers with complex cross section. IEEE Transactions on Microwave Theory and Techniques 2002;50(10):2339-2345.

[35] Sumant PS, Cangellaris AC, Aluru NR. A conformal mapping-based approach for fast two-dimensional FEM electrostatic analysis of MEMS devices. International Journal of Numerical Modelling: Electronic Networks, Devices and Fields 2011;24(2):194-206.

[36] Calixto WP, da Mota JC, de Alvarenga BP. Methodology for the reduction of parameters in the inverse transformation of Schwarz-Christoffel applied to electromagnetic devices with axial geometry. International Journal of Numerical Modelling: Electronic Networks, Devices and Fields 2011;24(6):568-582.

[37] Bao X, Bao J, Ocket I, Liu S, Schreurs D, Kil D, et al. A Simplified Dielectric Material Characterization Algorithm for Both Liquids and Solids. IEEE Transactions on Electromagnetic Compatibility 2018;61(5):1639-1646.

[38] Hiebel M. Fundamentals of vector network analysis. Rohde \& Schwarz; 2007.

[39] Marks RB. A multiline method of network analyzer calibration. IEEE Transactions on Microwave Theory and Techniques 1991;39(7):1205-1215.

[40] Marks RB, Williams DF. A general waveguide circuit theory. Journal of research of the National Institute of Standards and Technology 1992;97(5):533.

[41] Bao X, Liu S, Ocket I, Bao J, Kil D, Zhang M, et al. A Multiline Multimaterial Calibration Method for Liquid Characterization. IEEE Microwave and Wireless Components Letters 2018;28(8):732-734.

[42] Crupi G, Schreurs D. Microwave de-embedding: from theory to applications. Academic Press; 2013.

[43] Ellison W. Permittivity of pure water, at standard atmospheric pressure, over the frequency range $0-25 \mathrm{THz}$ and the temperature range 0-100 C. Journal of Physical and Chemical Reference Data 2007;36(1):1-18.

[44] Bao J, Markovic T, Brancato L, Kil D, Ocket I, Puers R, et al. Novel Fabrication Process for Integration of Microwave Sensors in Microfluidic Channels. Micromachines 2020;11(3):320. 
[45] Heinrich W. Quasi-TEM description of MMIC coplanar lines including conductor-loss effects. IEEE Transactions on Microwave Theory and Techniques Jan 1993;41(1):45-52.

[46] Bao X, Ocket I, Bao J, Liu Z, Puers B, Schreurs DMP, et al. Modeling of Coplanar Interdigital Capacitor for Microwave Microfluidic Application. IEEE Transactions on Microwave Theory and Techniques 2019;67(7):2674-2683.

[47] Abramowitz M. Handbook of Mathematical Functions with Formulas, Graphs, and Mathematical Tables, NBS. Applied Math Series 1964;55:232. 